\title{
INTERVENCIONES MUSICOTERAPÉUTICAS PARA LA ACTIVACIÓN DEL SISTEMA RETICULAR
}

\section{MUSIC THERAPEUTIC INTERVENTIONS FOR RETICULAR ACTIVATING SYSTEM}

\author{
Veronika Diaz Abrahan* ${ }^{*}$ Melisa Fischer ${ }^{* *}$ y Nadia Justel ${ }^{* * *}$
}

\author{
*Licenciada en Musicoterapia y Doctoranda en Neurociencias. Becaria del Consejo Nacional de Investigaciones \\ Científicas y Técnicas (CONICET). E-Mail: abrahanveronika@gmail.com \\ ** Licenciada en Musicoterapia. Musicoterapeuta de la Residencia para Adultos Mayores Los Robles (Adrogué, Prov. \\ de Buenos Aires), del Centro Educativo Terapéutico para Niños con Autismo (Banfield, Prov. de Buenos Aires) y de la \\ Escuela de Educación Especial LIWEN. E-Mail: melisadfischer@hotmail.com \\ ***Doctora en Psicología. Miembro de la Carrera del Investigador Científico del Consejo Nacional de Investigaciones \\ Científicas y Técnicas (CONICET). E-Mail: nadiajustel@gmail.com \\ Laboratorio de Psicología Experimental y Aplicada (PSEA). \\ Instituto de Investigaciones Médicas (IDIM). \\ Consejo Nacional de Investigaciones Científicas y Técnicas (CONICET) - Universidad de Buenos Aires (UBA). \\ Combatientes de Malvinas 3150, Planta Baja, $2^{\circ}$ Cuerpo. \\ (1427) Ciudad Autónoma de Buenos Aires. República Argentina.
}

\section{REsUmEN}

La musicoterapia es una disciplina que progresivamente está ampliando su campo de abordaje. Dentro de las nuevas intervenciones se pueden mencionar las implementadas en pacientes con estado alterado de conciencia y cuadros patológicos, producto de lesiones neuronales adquiridas que involucran al sistema reticular. La formación reticular es una red neuronal que establece conexiones con gran parte del sistema nervioso, regulando el alerta general, la dirección de la atención y la transición entre sueño y vigilia. A partir de esto, el trabajo que se informa está centrado en una descripción general de los diferentes estados alterados de conciencia y las intervenciones de una disciplina científica, con técnicas no invasivas que utiliza el sonido y sus elementos específicamente en el diagnóstico y abordaje terapéutico de pacientes en estado de mínima conciencia y su efecto en la formación reticular, teniendo como objetivo principal la rehabilitación del paciente y el aumento de sus niveles de alerta. Las técnicas en musicoterapia se basan en los resultados positivos del efecto de ciertos parámetros sonoros tales como la melodía, los aspectos temporales, la armonía, sobre indicadores fisiológicos (frecuencia respiratoria y cardíaca, conductancia de la piel, niveles de cortisol, etc.), que proporcionan un marco organizativo. A través de dicho marco las personas pueden responder e interactuar por medio de las propuestas musicales, determinadas por características, tales como la simplicidad, la utilización de la voz sin acompañamiento, improvisando vocales, melodías repetitivas, música de baja densidad cronométrica unida a la velocidad respiratoria del paciente y que pertenezca a su entorno familiar.

Palabras clave: Musicoterapia; Sistema reticular; Estado de mínima conciencia; Técnicas; Intervenciones. 


\section{ABSTRACT}

Music-therapy is a scientific discipline that in the last years has extended its work fields, to multiple populations and pathologies. Among the new interventions is the one related to altered conscious states, which are pathological disorders product of acquired neuronal lesions that involved the reticular system. Currently, there are different and varied models music-therapy that works with patients with altered consciousness states and the pathologies associated to those disorders. Parallel to the cognitive rehabilitation a branch emerged called neurological music-therapy, which is based in the neuroscientific model of musical production and perception and it is defined as the therapeutic application of music and theirs discursive elements in people who suffered motor, cognitive and sensorial deficits caused by neurological diseases. The reticular system is a neuronal network, known as a complex area due to its anatomy and functional heterogeneity. This network stablish connections with a large portion of the nervous system, acts as a filter system as it is responsible for separating incoming stimuli, discriminating between those relevant and not relevant, and it regulates the general alert, direction of attention, transition between awake, and sleep states. The lesions and deficits that involved the reticular system had been a point of scientific interest for many years. There are two main components related to conscience: the arousal or wakefulness (be aware) and the awareness (the content of conscience). The arousal is considered a preparation state to action that consists in primitive behaviors to the environment, i.e. involuntary behaviors. To maintain the arousal intervenes the ascendant reticular system. While awareness involved a complex process, a cognitive elaboration of thought. Such elaboration encompasses the process of information that is received through the senses and environmental demands. When these processes are interrupted because of traumas or anomalies could generated a coma or states where the content of conscious are affected. After the brain injury, the patients are in a coma state and from there they could emerged with different long-term results: Post-traumatic amnesia, Minimal conscious state, vegetative state or death. The music-therapy techniques used with this population are descripted in this work from a neurological model, which is based in empirical evidence. This evidence is result of the effect of specific sound parameters (as melody, rhythm, harmony) upon physiological indicators (as heart rate, respiratory frequency, skin conductance, cortisol levels, among others), as well as the use of familiar music for the patient, detecting physiological indicators during musical experiences. The musictherapy techniques provide and motivate an organizational framework through which patients with severe disorders of their expressive and receptive linguistic abilities could respond and interact through musical applications. These techniques are possible because music is an innate ability of all human beings, also because the auditive system is the first sense that develops in the intrauterine level. The characteristics of music-therapy techniques are defined for simplicity, the use of the voice, where repetitive melodies are improvised, without breaking the laws of musical expectation, with low chronometric density music; this type of music go along with the respiratory speed of the patient. Also the musical history of the patient is taken into account to enable a familiar environment. With this background, the current work focuses in a general description of the different conscious states, the implication of reticular system and the interventions of music-therapy as a scientific discipline that offers non-invasive techniques that used the sound and their elements to test, to diagnostic and to treat patients in a minimal state of conscious. This technique has the ultimate goal of rehabilitate the cognitive functions and enhance the alert levels, besides providing a reinforcement for the interdisciplinary approach.

Key words: Music-therapy; Reticular system; Disorders of consciousness; Technique; Intervention.

\section{INTRODUCCIÓN}

La formación reticular es una red conformada por grupos de escasas neuronas que poseen conexiones indefinidas e involucra gran parte del sistema nervioso. Se ubica dentro del área tegmental, en el centro del tronco 
encefálico y vincula regiones que van desde la sustancia gris de la médula espinal cervical hasta regiones laterales del hipotálamo y núcleos a lo largo de la línea media del tálamo (Purves et al., 2007). Las extensiones de la red del tronco encefálico (distribuidas en las zonas paramedial, medial y lateral) reciben información sensitiva y sensorial tanto de nervios craneanos como de la médula espinal. Esta información luego se propaga a través de impulsos por diversas áreas del sistema nervioso, como la médula espinal y la corteza cerebral (Alcaraz Romero, 2001).

Esta formación es reconocida como un área sumamente compleja en cuanto a su anatomía y su heterogeneidad funcional (Purves et al., 2007). Actúa como un sistema de filtro, ya que es la encargada de separar los estímulos entrantes, discriminando entre aquellos relevantes y no relevantes, funciona además como despertador del sistema nervioso ya que mantiene el estado de vigilia (Curtis, Barnes, Schnek \& Massarini, 2000) a través del Sistema Activador Reticular Ascendente (SARA - Aguilar Morales, 2011). También participa en la sensibilidad somática y visceral, en el control del sistema nervioso automático del sistema endócrino, del sueño y en el nivel de activación (arousal) de la atención (Aguilar Morales, 2011). En este sentido, hace de agente de conexión entre lo sensitivo y lo motor; sin embargo, es junto a la extensión talámica que logra ser un sistema pre-motor que modula los estados de conciencia y luego en retroalimentación organizan las respuestas motoras. Entonces, el SARA regula el alerta general, la dirección de la atención, siendo el despertador del sistema nervioso y regulando la transición entre sueño y vigilia.

Los cuadros patológicos producto de lesiones neuronales que involucran al sistema reticular han sido tema de interés por muchos años. Dentro del conjunto de las últimas investigaciones relativas a la temática de los estudios sobre el uso de la música en los pacientes con estados alterados de conciencia se encuentran las de Magee (2007), Peretz y Zatorre (2005) y Rollnik y Altenmüller (2014). El procesamiento de la música en pacientes con lesiones cerebrales severas con trastornos de la conciencia ha sido un campo de interés que abarca las disciplinas de las Neurociencias, la Medicina, las Artes, las Humanidades y específicamente la Musicoterapia, disciplina científica que se basa en el uso de la música y sus elementos con fines terapéuticos (Bruscia, 1998). Como existen pocas opciones de tratamiento, las técnicas musicoterapéuticas no invasivas podrían constituir una estrategia prometedora para estimular la plasticidad del cerebro y mejorar la recuperación de la conciencia (Altenmüller \& Schlaug, 2013).

Se presenta una visión general de los cuadros patológicos que implican la alteración en el sistema reticular, conocidos como estados alterados de conciencia y las intervenciones terapéuticas de una disciplina que crece día a día dentro de la temática: la Musicoterapia.

\section{Estados ALTERADOS DE CONCIENCIA}

Luego de la lesión cerebral adquirida, los pacientes ingresan en estado de coma y pueden emerger de allí con severos resultados a largo plazo, por ejemplo con Amnesia PostTraumática (APT), Estado de Mínima Conciencia (EMC), Estado Vegetativo (EV) o la muerte (Baker \& Tamplin, 2006). Antes de dar cuenta de cada uno de estos estados, es importante describir dos componentes principales con respecto a la conciencia y distinguidos por Plum y Posner, el nivel de alerta o estar consciente (arousal o wakefulness) y el contenido de la conciencia o ser consciente (awareness - Noé Sebastián et al., 2012).

El nivel de alerta (arousal) es considerado un estado de preparación para la acción que consiste en respuestas primitivas al medioambiente, es decir, respuestas involuntarias. Para producir y mantener el arousal interviene el SAR (Baker \& Tamplin, 2006). El contenido de la conciencia (awareness) implica un proceso más complejo, una elaboración cognitiva del pensamiento (Tamplin, 2000). Según Baker, dicha elaboración involucra el procesamiento de la información recibida por medio de los sentidos y relacionada con las demandas del medioambiente. Cuando estos procesos son interrumpidos por 
traumas o anomalías se pueden generar coma o estados en los que el contenido de la conciencia se encuentre perjudicado (Baker \& Tamplin, 2006; Davis \& Gimenez, 2003).

\section{SíNDROME DE BLOQUEAMIENTO}

Aunque no esté presentado como un estado de salida del coma, para los profesionales que trabajan con esta población es relevante conocer sus características, ya que puede ser confundido fácilmente con el estado vegetativo (EV), porque en ambas condiciones hay una falta de respuesta física al ambiente. Sin embargo, a diferencia del paciente en $\mathrm{EV}$, el paciente en estado de bloqueamiento puede indicar conciencia (Baker \& Tamplin, 2006). Este síndrome suele ser resultado de una patología del tronco cerebral que altera el control voluntario del movimiento, sin abolir el estado de vigilia o de conciencia. Es una condición extraña en la que los pacientes están paralizados pero conscientes y por lo general, se pueden comunicar utilizando movimientos de los ojos o párpados (Giancino \& Zasler, 1995; Royal College of Physicians, 2013). El Congreso Americano de Medicina de Rehabilitación (1995) lo caracterizó por la presencia de apertura sostenida de los ojos, afonía o hipofonía grave, cuadriplejia o tetraparesia, funcionamiento cognitivo conservado y un código primario y elemental de comunicación usando los movimientos oculares verticales o parpadeo (Schnakers, 2009). Estos pacientes tienen necesidades físicas y emocionales altas ya que las habilidades cognitivas, como la percepción y conciencia de sus impedimentos, pueden estar relativamente intactas y conducir a problemas de ajuste emocional en el comienzo del proceso de recuperación (Baker \& Tamplin, 2006).

\section{Amnesia Post-Traumática (APT)}

La transición del estado de coma hasta el de conciencia máxima sigue un proceso por etapas. Se denomina amnesia post-traumática a la fase que atraviesan los pacientes que emergen del coma y se caracteriza por una alteración cognitiva generalizada con un mayor nivel de respuesta, pero permanecen confundidos (Artiola et al., 1980; Forrester, Encel \& Geffen, 1994; Gasquoine, 1991; Geffen, Encel \& Forrester, 1991; Ponsford, Sloan \& Snow, 1995). Se pueden distinguir las siguientes características en este tipo de pacientes: limitada conciencia de las circunstancias, amnesia retrógrada y anterógrada, fatiga física como cognitiva y agitación.

\section{Estado de Mínima Conciencia (EMC)}

Un equipo de profesionales de distintas especialidades reunido entre 1995 y 1996 en Estados Unidos (Aspen Neurobehavioral Conference Work-group) propuso sustituir el nombre de estado de mínima respuesta por el de estado de mínima conciencia con el objetivo de resaltar la voluntariedad de las respuestas. En 2002 se definieron los siguientes criterios de EMC: respuestas a órdenes simples, respuestas verbales o no verbales independientemente de su grado de acierto, verbalización inteligible, conductas dirigidas a un fin, incluyendo movimientos o respuestas congruentes con estímulos relevantes. Además, se definieron los criterios de emergencia de dicho estado: evidencia de comunicación funcional y de uso funcional de los objetos (Noé Sebastián et al., 2012). En este sentido, Wainsztein y Leiguarda (2005) y la Royal College of Physicians coinciden con estos criterios e indican que para diagnosticar el EMC las respuestas pueden ser inconsistentes pero reproducibles, por encima del nivel de la conducta reflexiva, lo que indica cierto grado de interacción con su entorno y la presencia del ciclo sueño-vigilia.

\section{Estado Vegetativo (EV)}

El cuadro de estado vegetativo es consecuencia de una lesión cerebral adquirida severa y se define como un estado de arreactividad completa de uno mismo y del entorno, acompañado de una preservación completa o 
parcial del ritmo de sueño-vigilia y de las funciones troncoencefálicas e hipotalámicas (Noé Sebastián et al., 2012). Además, no hay expresión o comprensión del lenguaje. Estos pacientes manifiestan desvelo intermitente, presentan incontinencia del intestino y vejiga, con una preservación variable de los reflejos de pares craneales (pupilar, oculocefálico, corneal, vestíbulo-ocular y mordaza) y reflejos espinales. El pronóstico de vida de estos pacientes es pobre (Baker \& Tamplin, 2006; Bodard, Laureys \& Gosseries, 2013).

\section{Estado Vegetativo Persistente (EVP)}

Se considera estado vegetativo persistente cuando la duración del cuadro clínico correspondiente al EV se prolonga más de 3 meses, si la etiología es hipoxicoisquémica, metabólica y congénita, y más de 12 meses cuando la etiología es postraumática (Hodelín-Tablada, 2002). Transcurrido ese tiempo se propuso por primera vez el polémico adjetivo con connotaciones pronósticas de permanente (Noé Sebastián et al., 2012).

"Una conducta consciente normal requiere de ambos componentes, la capacidad y el contenido de la conciencia. (...) En los pacientes en EVP la capacidad está preservada, mientras que el contenido de la conciencia está aparentemente ausente" (Cabrera-Lima, 1999, p. 1105).

\section{COMA}

El estado de coma se define por la falta de apertura de los ojos, por no obedecer a comandos u órdenes y por no pronunciar palabras comprensibles (Ponsford et al., 1995). Es un período extendido de inconciencia, un estado sin respuestas, donde el individuo no muestra comportamientos o movimientos voluntarios (Plum \& Posner, 1980).

Esta pérdida de conciencia sobre sí mismo y sobre el medio ambiente que lo rodea, además de la falta de ciclo de sueño-vigilia (Ro- yal College of Physicians, 2013) puede durar minutos o años y se debe a la interferencia en el funcionamiento del córtex cerebral que afecta al contenido de la conciencia (awareness) y / o a la interferencia en el funcionamiento de las estructuras que despiertan el SAR, perturbando así al nivel de alerta (arou$s a l$ ). El grado o nivel de la pérdida dependerá de la severidad de la lesión y de su localización (Baker \& Tamplin, 2006).

El estado de coma ocurre como consecuencia de la combinación del daño cerebral permanente y de la disfunción temporaria del cerebro. Debido a la lesión puede ocurrir una contusión cerebral que daña el tejido cerebral y además puede inflamarse, por lo que temporalmente deja de realizar sus operaciones para así conservar la energía y sanar. El tronco cerebral mantiene en funcionamiento a los órganos vitales, mientras la persona descansa y se recupera. A medida que el cerebro comienza a desinflamarse, cada vez más partes del cerebro lesionado comienzan a activarse, así poco a poco la persona comienza a responder a estímulos ambientales (Baker \& Tamplin, 2006; García, Suárez, Dávalos \& Villagómez, 2013).

En todos los estados descriptos anteriormente la conciencia está alterada en mayor o menor medida, en lo que respecta al nivel de alerta, el estar consciente o al contenido de la conciencia, el ser consciente. Una de las diferencias fue presentada, entre otras cuestiones más profundas, en el orden en que se le dio, de mayor a menor, según el nivel de conciencia de cada estado. Dicha información es importante para los profesionales musicoterapeutas que trabajan con esta población, para conocer sus características y para diferenciarlos, en una búsqueda constante de potenciales a trabajar desde la disciplina.

\section{ABORDAJE MUSICOTERAPÉUTICO EN LOS ESTADOS ALTERADOS DE CONCIENCIA}

La Federación Mundial de Musicoterapia define a la disciplina musicoterapéutica como: 
"El uso de la música y / o sus elementos musicales (sonido, ritmo, melodía y armonía) realizado por un musicoterapeuta calificado con un paciente o grupo, es un proceso creado para facilitar, promover la comunicación, las relaciones, el aprendizaje, el movimiento, la expresión, la organización y otros objetivos terapéuticos relevantes, para así satisfacer las necesidades físicas, emocionales, mentales, sociales y cognitivas. La Musicoterapia tiene como fin desarrollar potenciales y / o restaurar las funciones del individuo de manera tal que este pueda lograr una mejor integración intra y / o interpersonal y consecuentemente una mejor calidad de vida a través de la prevención, rehabilitación y tratamiento" (World Federation of Music Therapy, 2011, p. 1).

La Musicoterapia es una disciplina que presenta varios campos de abordaje. Actualmente existen diferentes modelos que intervienen en pacientes con estados alterados de conciencia. Por un lado se puede mencionar el Modelo Benenzon, el cual se trata de una aproximación humanista que parte de premisas de modelos dinámicos de intervención terapéutica, la teoría de la comunicación humana y otros ámbitos del conocimiento relacionados, como por ejemplo con aspectos psicológicos de Jung, Freud, Winnicot, entre otros (Benenzon, 2011). Por otra parte, y en paralelo a los desarrollos de la rehabilitación cognitiva ha surgido una rama de la disciplina llamada Musicoterapia Neurológica (MTN) basada en el modelo neurocientífico de percepción y producción musical y definida como la aplicación terapéutica de la música en personas que padecen déficit cognitivos, sensoriales y motores causados por enfermedades neurológicas (Thaut \& Hoemberg, 2014), el cual se ajusta adecuadamente al marco del trabajo que se informa.

La musicoterapeuta Wendy Magee (2007) justifica el uso de la música como un medio de tratamiento en la neuro-rehabilitación, específicamente en el diagnóstico y abordaje terapéutico del EV y del EMC. En este sentido, expone que hay varias razones simples de por qué la música puede ser un medio de tratamiento efectivo para pacientes en estados de baja conciencia, quienes pueden tener compromisos severos en sus facultades lingüísticas expresivas y receptivas y pueden experimentar una gama de respuestas emocionales a su situación inmediata y permanente. En primer lugar, toda la comunicación temprana se basa en parámetros musicales tales como el pulso, la dinámica, el contorno melódico, la articulación, el timing y el fraseo (Español, 2014; Papousek, 1996; Trevarthen, 1999, 2002).

Las propuestas terapéuticas que involucran a la música proporcionan un marco organizativo a través del cual las personas pueden responder e interactuar. Esto se debe a que mucho antes del desarrollo del lenguaje, los bebés comunican sus estados emocionales inmediatos mediante vocalizaciones, siendo la música una habilidad innata en todos los seres humanos. Además de ser un medio social de gran poder intercultural durante toda la vida (Blacking, 1976), es importante tener en cuenta que el sistema auditivo es el primero que se desarrolla durante el crecimiento intrauterino, con lo cual los vínculos tempranos se propician a través de estímulos sonoros entre la madre y el bebé (Shifres, 2014). Por otra parte la música provoca y transmite estados emocionales (Eickhoff et al., 2008; Sloboda, 1991a, 1991b), teniendo en cuenta el compromiso neurocognitivo que implica la simple percepción de un estímulo sonoro, estimulando los circuitos neuronales implicados en el procesamiento emocional (Koelsh, 2010). Esto ha despertado un gran interés en los investigadores, conformando un cuerpo creciente de evidencias que sostienen que la música contribuye a la neuroplasticidad, permitiendo así efectuar las conexiones entre los centros del cerebro dañados y saludables en poblaciones con daño cerebral adquirido (Abrahan \& Justel, 2015; Magee, 2005; O'Callaghan, 1999).

INTERVENCIONES MUSICOTERAPÉUTICAS PARA PACIENTES CON ESTADO ALTERADO DE CONCIENCIA

En los pacientes con desórdenes prolongados de conciencia, la Musicoterapia proporciona principalmente, una evaluación detallada de su estado multisensorial y así 
contribuye aportando información con procedimientos diagnósticos propios, para la evaluación interdisciplinaria de la conciencia y la planificación del proceso de su rehabilitación, estableciendo objetivos comunes que satisfagan sus necesidades (Thaut \& Hoemberg, 2014).

Existe evidencia sobre la efectividad del tratamiento musicoterapéutico con resultados positivos, a través del aumento de estados de relajación y la reducción de la agitación psicomotora, utilizando diferentes técnicas que tienen en cuenta el efecto en la reducción de la activación de estructuras como el hipotálamo, la glándula pituitaria y adrenal, conocidas como el eje HPA (Crawford, Hogan \& Silverman, 2013; Magee \& O’Kelly, 2014) así como también la disminución de cortisol y b-Endorfinas luego de la implementación de la técnica de imaginería guiada con música (McKinney, Antoni, Kumar, Tims \& Mc Cabe, 1997). Para el logro de dichas mejorías, el musicoterapeuta debe establecer en primer lugar contacto con el paciente. Una de las vías es lograr establecer una sincronización de la respiración de ambos. Luego puede acompasar la respiración del paciente con el fraseo de la música y esto afectaría los patrones respiratorios haciéndolos más consistentes, lentos y profundos. Podrían considerarse como indicadores del aumento de la conciencia del medio ambiente que un paciente gire hacia el origen de la música tratando de localizar la fuente, o que abra los ojos durante el canto o el inicio del mismo teniendo conciencia del sonido, o los abra cuando la música cesa, indicando conciencia sobre la procedencia de los estímulos musicales o del cambio de estímulo (Tamplin, 2000). Es importante que el terapeuta esté especialmente atento a los diversos indicadores ya que el paciente podría presentar espasmos o movimientos repetitivos debido al dolor o agitación y esto no sería una respuesta a la terapia sino que se trataría de un comportamiento reflexivo (Baker \& Tamplin, 2006; Formisano et al., 2001). Hay otras formas de establecer contacto, incluso incorporando a la familia, tanto para conocer sus gustos musicales como para que ella intervenga cantando junto a él, o aseso- rada por el musicoterapeuta. Al conocer qué tipo de temas le agradan, el especialista puede interpretarlos con su guitarra, y aunque el paciente no parezca responder físicamente a esto, a la larga hay casos que relatan recordarlo. Pueden cantarle, hacerle escuchar esas grabaciones, en fin, hay varias formas de acercamiento, también se puede consultar bibliografía sobre el tema.

Cuando un paciente comienza a salir del coma, las intervenciones musicoterapeuticas pueden focalizarse en desarrollar una forma consistente de comunicación. Típicamente al principio se utilizan gestos como parpadeos, afirmaciones con la cabeza, pulgares arriba o abajo, movimiento de dedo o de pie que son utilizados como respuestas hacia las preguntas de sí o no. La evaluación del conocimiento y habilidades de los pacientes para seguir direcciones sencillas puede ser alcanzada a través de actividades musicales simples, como solicitarle que agite campanas, o rasguñe con los dedos un tambor. Cabe reiterar que los profesionales deben estar atentos para determinar si la falta de respuesta es un indicador de bajo nivel de alerta o, por el contrario, de impedimentos físicos residuales, ya que las opciones de futuros tratamientos deben ser tomadas en base a esta evaluación (Baker \& Tamplin, 2006).

Es importante hablar con los pacientes en estado de coma como si ellos fuesen capaces de oír y comprender (LaPuma, Schiedermayer, Culyas \& Siegler, 1988). Los reportes anecdóticos de pacientes que salieron del estado de coma indican cierto recuerdo de los eventos que ocurrieron mientras estaban en ese estado (Ansdell, 1995).

Con respecto a las herramientas propias de los musicoterapeutas, la música editada ofrece un estímulo estandarizado, aunque el uso de música en vivo le brinda a los profesionales mayores ventajas que tienen que ver con la posibilidad de improvisar, generar cambios en relación a lo que esté sucediendo en ese momento con el paciente, adaptándose por ejemplo a los cambios de atención o de excitación, mencionados anteriormente $\left(\mathrm{O}^{\prime}\right.$ Kelly et al., 2013; Vogl, Heine, Steinhoff, Weiss \& Tucek, 2015). 
Para la evaluación durante el proceso musicoterapéutico, existe un protocolo desarrollado por Magee, llamado MATADOC basado en evidencia. Esta herramienta sistemática es una medida derivada de la clínica e impulsada por la investigación, que utiliza principalmente experiencias musicales en vivo para evaluar la conciencia y la capacidad de respuesta en pacientes con desórdenes prolongados de la conciencia. Permite la observación del comportamiento antes y después del estímulo, en base a la modalidad auditiva mediante una gran gama de estímulos sonoros tanto verbales como no verbales, proporcionando una evaluación más sensible de la capacidad de respuesta auditiva. Estos estímulos pueden incluir acciones como llamar al paciente por su nombre, golpear bloques de madera o aplaudir cerca de las orejas del paciente, soplar un silbato, sacudir cascabeles, tocar campanas, o reproducir grabaciones de música o voces familiares que dan mensajes verbales. Finalmente el protocolo proporciona una línea de base de los comportamientos del paciente en reposo, con la relevancia que ello tiene para la comparación posterior (Magee \& O'Kelly, 2014).

INTERVENCIONES MUSICOTERAPÉUTICAS EN PACIENTES CON AMNESIA POSTRAUMÁTICA (APT)

Para estos pacientes el medio ambiente se les presenta desconocido y amenazador, ya que no poseen la habilidad cognitiva para darle sentido a lo que los rodea, por lo que es importante que se sientan cuidados y tranquilos. Esta confusión puede resultar en una elevación de sus niveles de agitación, por ello necesitan disminuir los mismos y aumentar la orientación hacia el medio ambiente. Los niveles máximos de recuperación se alcanzan cuando el paciente está relajado y descansado (Ponsford et al., 1995), por lo tanto se recomienda un mínimo de estimulación. En este sentido muchos considerarían a priori que la Musicoterapia no sería indicada debido a que el potencial estimulador de la música puede generar agitación en el paciente. Sin embargo, hay diversos estudios que demuestran que los niveles de arousal pueden disminuir con la Musicoterapia y esto podría ser utilizado para el tratamiento de estos pacientes induciendo la relajación (Chanda \& Levitin, 2013).

Para disminuir la agitación y la conducta autolesiva se utilizan estrategias que habiliten a los pacientes a moverse al ritmo de la música, permitiéndoles organizar sus movimientos, que de lo contrario son agitados. La sincronización no implica esfuerzos para el paciente y al utilizar música que le resulta familiar, disminuye su ansiedad. Otro objetivo es orientar al paciente en tiempo y espacio, para ello es recomendable que escuche música que le resulte familiar (Baker, 2002), ya que le permite reconocer elementos de su medio ambiente y por ende tiende a participar más en el mismo. Además, escuchar este tipo de música puede colaborar disminuyendo el nivel de angustia (Baker \& Tamplin, 2006; Rollnik \& Altenmüller, 2014).

Dentro de las intervenciones musicoterapéuticas la selección de los estímulos musicales se realiza de acuerdo a la colección musical del paciente, solicitando ayuda a familiares o amigos cercanos. Estos estímulos no deberían ser activantes, sino más bien relajantes con una valencia positiva, debido a que este tipo de estimulación disminuye los niveles de activación / arousal en el paciente (Chanda \& Levitin, 2013). Algunas de las estrategias específicas que utiliza el músicoterapeuta son: contarle que él va a cantar con el paciente, alentarlo a que se relaje y escuche la música, cantar con el paciente con voz calmada (O'Kelly \& Magee, 2013a).

\section{INTERVENCIONES MUSICOTERAPÉUTICAS CON PA-} CIENTE CON ESTADO DE MÍNIMA CONCIENCIA (EMC)

Las necesidades de estos pacientes tienen un nivel de respuesta leve y se basan en adquirir un método de comunicación, por lo que los objetivos del terapeuta estarán orientados a identificar un posible medio de comunicación con el paciente, ya sea verbal o gestual (Aldridge, Gustorf \& Hannich, 1990; Baker \& Tamplin, 2006), así como también estimular el alerta, recuperar el ciclo de vigilia, facili- 
tar respuestas significativas y la orientación temporoespacial (Thaut \& Hoemberg, 2014).

Dentro del modelo de Musicoterapia Neurológica existe una técnica especializada denominada entrenamiento musical sensorial de la orientación, la cual se focaliza en la estimulacion sensorial, el arousal, la orientación y vigilia a través del canto de canciones familiares las cuales deben ser simples, predecibles, repetibles y a capella (Thaut, 2005). También se encuentra la improvisación desde la voz, teniendo en cuenta que las frases suban y bajen con el patrón de respiración del paciente. Estas intervenciones serán planificadas según las necesidades del paciente y en colaboración con el equipo interdisciplinario (O’Kelly \& Magee, 2013b).

En el caso de los pacientes en EMC con respuesta moderada, las intervenciones considerarán además su necesidad de tener un método de interacción con otros; se evaluará la comprensión de su lenguaje y su capacidad para seguir los pasos de instrucciones simples. Además de la improvisación vocal y del canto de canciones, las intervenciones apuntarán a que el paciente pueda participar de una actividad musical sencilla, ubicando los instrumentos de manera que se facilite al máximo su acceso para su manipulación o fijándolos a alguna parte de su cuerpo cuando el paciente no pueda tomarlos, sirviéndole de guía y colaborando con él para promover su activación neurológica (Baker \& Tamplin, 2006; Daveson, Magee, Crewe, Beaumont \& Kenealy, 2007).

\section{INTERVENCIONES MUSICOTERAPÉUTICAS CON PA- CIENTES EN ESTADO VegETATIVO (EV)}

El tratamiento musicoterapéutico en este tipo de población es cuestionado por los administradores de los hospitales, ya que desde su perspectiva ellos no responden a la estimulación sensorial. Otra consideración es si los pacientes con EV son o no permanentes. Según Baker y Tamplin (2006), algunos pacientes no se recuperan suficientemente para demostrar conciencia y el proceso terapéutico puede ser lento y limitado.
Las necesidades de estos pacientes son similares a las de los que están en estado de coma ya que responden en un nivel similar, pero se distinguen dos condiciones: los ciclos de sueño-vigilia y la longitud del tiempo de evolución. Con estos pacientes, el musicoterapeuta puede tener un rol muy importante ya que necesitan el contacto humano de modo regular y significativo, así como sostener los niveles de estimulación (Baker \& Tamplin, 2006). Wilson, Powell, Brock y Thwaites (1996) indican que los mayores cambios en el comportamiento fueron observados luego de la presentación de estímulos significativos para el paciente, incrementando sus niveles de alerta, como por ejemplo con canciones o sonidos de su entorno familiar / social.

Con respecto a la importancia de los estímulos auditivos con carga emocional, un estudio que evaluó las diferencias en la activación cerebral de pacientes en EV y EMC, en respuesta a escuchar su nombre dicho por una voz familiar, reveló que escucharlo es el estímulo más poderoso para acceder a la conciencia, ya que activa la corteza cerebral más ampliamente en comparación con otros estímulos emocionales. Las mayores respuestas se detectaron cuando el nombre propio era pronunciado por una voz familiar (por ejemplo, el hijo). Por lo tanto para detectar funciones cerebrales residuales en pacientes en EV y en EMC se puede realizar este tipo de intervención (Machado, 2007). Es importante tener en cuenta que suele ser problemático para los clínicos determinar si los comportamientos observados en estos pacientes son útiles, ya que muchas veces suelen sonreír, lagrimear, gruñir o en raras ocasiones, gemir o gritar, pero estos son comportamientos inconscientes, no-intencionales y sin propósito; por ello puede ser engañoso sugerir que estos indicarían conciencia y respuesta al ambiente (Giancino \& Zasler, 1995).

Para evaluar pacientes en EV o que están saliendo del coma se utilizan las siguientes escalas: Coma-Near Coma Scale (Rappaport, Dougherty \& Kelting, 1992), Sensory Stimulation Assesment Measure (Rader \& Ellis, 1989), Coma Recovery Scale (Giacino, Kezmarsky, DeLuca \& Cicerone, 1991) y Wes- 
tern Neuro Sensory Stimulation Profile (Ansell \& Keenan, 1989). Con estas escalas es posible reconocer y categorizar los comportamientos, resultando importante su administración para poder reducir conjeturas y establecer un comportamiento de base para medir el cambio. Para determinar si un paciente está respondiendo al tratamiento se evalúan el seguimiento de los ojos (ya que el primer signo de que está saliendo del EV es si logra fijar la mirada en determinado objeto) y las respuestas emocionales, aunque es difícil determinar la fiabilidad de las observaciones (Baker \& Tamplin, 2006).

Una de las intervenciones más comunes con estos pacientes es la estimulación o regulación sensorial, aunque no está rigurosamente estudiada. Esta forma de intervención se basa en la noción de que la función mental y la recuperación cortical son afectadas adversamente con la privación sensorial (Mitchell, Bradley, Welch \& Britton, 1990). Por este motivo se apunta a reducir los ruidos extraños y a monitorear la naturaleza y frecuencia de las intervenciones del equipo terapéutico, ya que a este tipo de pacientes es preferible exponerlos a una cantidad mínima de estimulación, evitando la sobrecarga sensorial y dándoles tiempo para que puedan descansar (Baker \& Tamplin, 2006).

La recuperación de los pacintes en EV usualmente es progresiva. Aquellos que han estado por un corto período de tiempo en EV pueden recuperar algunas de sus capacidades físicas y mentales. También hay casos que estuvieron en EVP durante más de un año y han logrado recuperar sus habilidades para pensar, comunicarse e independizarse (Baker \& Tamplin, 2006).

INTERVENCIONES MUSICOTERAPÉUTICAS PARA PACIENTES EN ESTADO DE COMA

Las neuronas corticales de los pacientes en este estado necesitan estimulación temprana para mantener el nivel de alerta y mejorar los resultados cognitivos a largo plazo después de una lesión cerebral. Los estudios indican que una intervención precoz (dentro de los prime- ros 6 meses luego de la lesión y con el paciente estabilizado médicamente) posibilita una recuperación máxima. Se utilizan programas de estimulación sensorial que promueven respuestas de los pacientes y un aumento de conciencia, permitiéndoles tener una interacción significativa con el medio ambiente. Entonces, se provee con estimulación significativa no amenazante, para alentar la orientación hacia el medio ambiente, y así disminuir el período de coma e incrementar el pronóstico de rehabilitación (Formisano et al., 2001; Magee \& O'Kelly, 2015; O'Kelly et al., 2013; Raglio et al., 2014).

Existen algunos puntos a tener en cuenta para realizar intervenciones musicoterapéuticas con estos pacientes. El terapeuta debe comunicarle al paciente que le cantará, utilizando frases lentas, claras, cortas y simples. Es importante observar su velocidad y ritmo respiratorio y usar ese tiempo para comenzar a cantar. Luego se irá modificando la intensidad y tiempo para elevar la respuesta del paciente. Es interesante además caminar alrededor de la cama para que localice el sonido (Baker \& Tamplin, 2006). La selección musical depende directamente de cómo se presente su estado de coma, edad, género y sus antecedentes musicales. Aquí los familiares y allegados tienen un papel primordial para brindar información, sin embargo, se evidenciaron diferencias entre lo que los familiares creen y lo que realmente le agrada o prefiere el paciente (Baker, 2001; Thaut \& Holmberg, 2014).

Por otro lado, las intervenciones que utilizan la improvisación musical pueden realizarse aisladamente o acompañada de música familiar la cual es editada para el paciente y se refiere a las canciones de su ambiente musical y a la información brindada por sus familiares en entrevistas iniciales (Gustorff, 2001; Kennelly \& Edwards, 1997; Tamplin, 2000). Es necesario simplificar la música para no sobreestimular al paciente, ya que este estado presenta dificultades para discriminar la información relevante de la que no lo es, por lo tanto es importante minimizar la complejidad de la información. Se recomienda entonces, el uso de la voz sin acompañamiento, cantar improvisando o cantar cancio- 
nes, como también el uso de música monofónica que además evita cambios exagerados en dinámica, tiempo y forma (Baker \& Tamplin, 2006; Boeseler, 2012).

También se recomienda utilizar música con componente vocal ya que al brindar un factor humano a la interacción musical los pacientes frecuentemente responden más al canto que al sonido de instrumentos (Aldridge et al., 1990). Además, la voz puede estimular centros emocionales en el cerebro a través de los cuales se pueden evocar memorias emocionales positivas (Rosenfeld \& Dun, 1999). Esto da cuenta de que la voz humana mantiene un potencial comunicativo y una significación emocional mayor que otros instrumentos musicales para pacientes en coma (Baker \& Tamplin, 2006).

Los resultados de algunos estudios demuestran que los pacientes parecen responder a ciertas características musicales como música lenta, unida a su velocidad respiratoria, melódica, repetitiva y tocada o cantada tranquilamente (Baker \& Tamplin, 2006; O'Kelly \& Magee, 2013a).

\section{Conclusiones generales}

La formación reticular tiene un rol crítico en la regulación de los niveles de alerta. A lo largo de esta revisión se abordaron las estructuras con las que se vincula, sus funciones y una vía terapéutica a través de la Musicoterapia cuyo objetivo principal es rehabilitar al paciente y aumentar sus niveles de alerta.

A partir del rastreo bibliográfico, cabe destacar la importancia de la colaboración de familiares y / o amigos de los pacientes en el proceso terapéutico, otorgando información y posibles recursos en todos los niveles de conciencia, para una adecuada evaluación y planificación del proceso terapéutico. Esto también es importante en el momento que el profesional realiza la selección musical ya que esta debe ser elegida de acuerdo al estado del paciente, edad, género y antecedentes musicales.

Para la selección de los estímulos musicales, todos los autores resaltan que con estos pacientes resultan más efectivos los que posean determinadas características, tales como simplicidad, la utilización de la voz sin acompañamiento, improvisando o cantando canciones, melodías repetitivas, música lenta unida a la velocidad respiratoria del paciente y que pertenezca a su entorno familiar. En caso de utilizar música editada, se debe tener en cuenta que ésta no sea muy compleja, para evitar la sobreestimulación.

Bruscia, importante referente en el campo de la Musicoterapia, alega que la intervención terapéutica se estructura en objetivos clínicos cuya meta es mejorar tanto la comprensión del paciente desde una perspectiva holística, como atender las necesidades de salud del individuo (1998).

Por lo tanto es importante considerar ante todo, el estado y las necesidades de los pacientes para elegir las técnicas a utilizar. Las diferencias entre los distintos niveles de conciencia serán sutiles y radicarán en los objetivos que cada profesional plantee. Por ejemplo, para un paciente en EMC (leve), el objetivo será generar posibles medios de comunicación y para un paciente en EMC (moderada), el objetivo será la interacción. Otro ejemplo que se desprende de la bibliografía es que las necesidades de los pacientes en EV y de aquellos que se encuentran en coma, son similares ya que responden al mismo nivel, por ende el objetivo será la regulación sensorial y sostener y aumentar en lo posible el nivel de alerta.

Según resultados reportados en las investigaciones de los últimos años es posible distinguir cambios fisiológicos en los pacientes frente a intervenciones musicoterapéuticas (Thaut \& Hoemberg, 2014). Dichos cambios dan cuenta de la activación cerebral, del aumento en la atención y en los niveles de alerta, en lo que está involucrado el SAR, que al recibir información sensitiva y sensorial actúa como filtro, seleccionando algunos estímulos y descartando otros, generando así un nivel de vigilia que se predispone a captar mejor los estímulos y que genera respuestas apropiadas al medio ambiente. He aquí la relevancia de este conocimiento para los músicoterapeutas quienes además, cuentan con una herramienta multimodal: la música que puesta en contexto 
y en relación con el paciente, sumada a la relevancia histórica, puede generar diversas emociones y asociaciones personales. Además, la música involucra todos los sentidos, no sólo el auditivo, por lo que la estimulación musical provoca diversas respuestas a distintos niveles, por ejemplo a nivel del sistema motor, kinestésico, fisiológico, etc. Los estímulos musicales en pacientes con estados alterados de conciencia acceden a zonas dañadas del cerebro a través de la neuroplasticidad generando progresivamente mayores conexiones desde áreas sin daño cerebral.

En los últimos años han aumentado las investigaciones relacionadas con la temática abordada (Abrahan \& Justel, 2015; Justel, O' Conor \& Rubinstein, 2015), presentando artículos que dan cuenta de la relevancia de la Musicoterapia para los pacientes con estados alterados de conciencia y haciendo hincapié en la importancia de sus aportes a los trabajos interdisciplinarios en diagnósticos diferenciales, investigación y tratamiento. Si bien el material teórico sobre la temática es escaso, pero con un prometedor crecimiento, se puede observar que la fuente de información y desarrollo teórico proviene de países extranjeros, observando así que Argentina queda afuera de la producción sobre la temática. La disciplina musicoterapéutica está en un momento de delimitación y especificidad profesional. Es de suma importancia mostrar las evidencias que justifiquen y conformen un cuerpo teórico propio para su inserción adecuada en otros ámbitos, como el de las neurociencias.

A partir de lo expuesto surge la necesidad de plantear aspectos específicos de la disciplina musicoterapéutica sobre los indicadores sonoros y comportamentales, observables por un terapeuta de la música, que pudiesen llegar a proporcionar información sobre el estado del paciente así como de sus funciones cognitivas luego de una lesión cerebral, y que finalmente permitan elaborar estrategias de rehabilitación. En este sentido, una investigacion aplicada sobre el efecto de ciertas técnicas, pertenecientes a la Musicoterapia Neurológica en población argentina parece ser un camino futuro posible.

\section{REFERENCIAS BIBLIOGRÁFICAS}

Abrahan, V. \& Justel, N. (2015). Improvisación musical. Una mirada compartida entre la musicoterapia y las neurociencias [Musical improvisation. A shared look between music therapy and neuroscience]. Revista Psicogente, 18(34), 372-384. http://doi.org/10.17081/psico.18.34. 512

Aguilar Morales, J.E. (2011). La estructura del sistema nervioso [The nervous system structure]. México: Asociación Oaxaqueña de Psicología A.C.

Alcaraz Romero, V.M. (2001). Estructura y función del sistema nervioso: Recepción sensorial y estados del organismo [Structure and function of the nervous system: sensory reception and states of the organism]. México, DF: El Manual Moderno.

Aldridge, D., Gustorff, D. \& Hannich, H.J. (1990). Where am I? Music therapy applied to coma patients. Journal of the Royal Society of Medicine 83(6), 345-346.

Altenmüller, E. \& Schlaug, G. (2013). Neurobiological aspects of neurologic music therapy. Music and Medicines Journal, 5, 210-216. http://doi.org/10.1177/1943862113505328

Ansdell, G. (1995). Music for life: Aspects of creative music therapy with adult clients. Londres: Jessica Kingsley Publishers.

Ansell, B. \& Keenan, J.E. (1989). The Western Neuro Sensory Stimulation Profile: A tool for assessing slow-to-recover head-injured patients. Archives of Physical Medicine and Rehabilitation, 70, 104-108.

Artiola, I., Fortuny, L., Briggs, M., Newcombe, F., Ratcliff, G. \& Thomas, C. (1980). Measuring the duration of PTA. Journal of Neurology, Neurosurgery and Phychiatry, 43, 377-379. 
Baker, F. (2001). Rationale for the effects of familiar music on agitation and orientation levels of people experiencing posttraumatic amnesia. Nordic Journal of Music Therapy, 10(1), 30-35.

Baker, F. (2002). The effects of live, taped and no music on people experiencing postraumatic amnesia. Journal of Music Therapy, 38(3), 170192.

Baker, F. \& Tamplin, J. (2006). Music therapy methods in neurorehabilitation. A clinician's manual. London \& Philadelphia: Jessica Kingsley Publishers.

Benenzon, R. (2011). Musicoterapia: De la teoría a la práctica. Nueva edición ampliada [Music therapy: From the theory to the practice. New expanded edition]. Buenos Aires: Paidós, Psicología, Psiquiatría, Psicoterapia.

Blacking, J. (1976). How musical is man? (2da. ed.). London: Faber and Faber.

Boeseler, K. (2012). Need-oriented, emotionalcommunicative dialogue in music therapy with coma /apallic syndrome patients in neurological early rehabilitation. En R. Jox (Ed.), Vegetative state: A paradigmatic problem of modern societies: Medical, ethical, legal and social perspectives on chronic disorders of consciousness (pp. 79-94). Münster: LIT Verlag.

Bodard, O., Laureys, S. \& Gosseries, O. (2013). Coma and disorders of consciousness: Scientific advances and practical considerations for clinicians. Seminars in Neurology Journal, 33, 83- 90. http://doi.org/10.1055/s-0033-1348965

Bruscia, K. (1998). Defining music therapy. Gilsum, NH: Barcelona Publishers.

Cabrera-Lima, A.V. (1999). Estado vegetativo persistente: Reflexiones sobre el marco ético de la medicina contemporánea [Persistent vegetative state: Reflections on bioethical framework of contemporary medicine]. Revista de Neurología, 28(11), 1104-1109.
Chanda, M. \& Levitin, D. (2013). The neurochemistry of music. Trends in Cognitive Sciences, 17(4), 179-193.

Crawford, I., Hogan, T. \& Silverman, M. (2013). Effects of music therapy on perception of stress, relaxation, mood, and side effects in patients on a solid organ transplant unit: A randomized effectiveness study. The Arts in Psychotherapy, 40(2), 224-229. http://doi.org/10.1016/j.aip.20 13.02.005

Curtis, H., Barnes, S., Schnek, A. \& Massarini, A. (2000). Biologia [Biology]. Argentina: Panamericana.

Daveson, B.A., Magee, W.L., Crewe, L., Beaumont, G. \& Kenealy, P. (2007). The music therapy assessment tool for low awareness states. International Journal of Therapy and Rehabilitation, 14, 544-549.

Davis, A.E. \& Gimenez, A. (2003). Cognitive-behavioral recovery in comatose patients following auditory sensory stimulation. Journal of Neuroscience Nursing, 35(4), 202-214.

Eickhoff, S.B., Dafotakis, M., Grefkes, C., Stöcker, T., Shah, N.J., Schnitzler, A. et al. (2008). fMRI reveals cognitive and emotional processing in a long-term comatose patient. Experimental Neurology, 214, 240-246. http://doi.org/10.10 16/j.expneurol.2008.08.007

Español, S. (2014). Psicologia de la música y del desarrollo [Music psychology and development]. Argentina: Paidós.

Formisano, R., Vinicola, V., Penta, F., Matteis, M., Brunelli, S. \& Weckel, J.W. (2001). Active music therapy in the rehabilitation of severe brain injuredpatients during coma recovery. Annali dell'Istituto Superiore di Sanita, 37, 627-630.

Forrester, G., Encel, J. \& Geffen, G. (1994). Measuring PTA: An historical review. Brain Injury, 8(2), 175-184. 
García, S., Suárez, S., Dávalos, E. \& Villagómez, A. (2013). Coma and impaired consciousness: An analytical review from an approach neuro. Part I. Journal of Medical and Surgical Specialties, 18, 56-68.

Gasquoine, P.G. (1991). Learning in PTA following extremely severe CHI. Brain Injury, 5, 167-175.

Geffen, G.M., Encel, J.S. \& Forrester, G.M. (1991). Stages of recovery during post traumatic amnesia and subsequent everyday memory deficits. Neuroreport: International Journal for the Rapid Communication of Research in Neuroscience, 2, 12-127.

Giacino, J.T., Kezmarsky, M.A., DeLuca, J. \& Cicerone, K.D. (1991). Monitoring rate of recovery to predict outcome in minimally responsive patients. Archives of Physical Medicine and Rehabilitation, 72, 897-901.

Giancino, J. \& Zasler, N. (1995). Outcomes after severe TBI: Coma, the vegetative state, and the minimally responsive state. Journal of Head Trauma Rehabilitation, 10, 40-56.

Gustorff, D. (2001). Beyond words: Music therapy with comatose patients an those with impaired consciousness in intensive care. En D. Aldridge, G. DiFranco, E. Ruud \& T. Wigram (Eds.), Music therapy in Europe. Roma: Instituto Nationale per lo Sviluppo Musicale nel Mezzogiorno.

Hodelín-Tablada, R. (2002). Estado vegetativo persistente. Paradigma de la discusión actual sobre alteraciones de la conciencia [Persistent vegetative state. Paradigm of current discussion on alterations of consciousness]. Revista de Neurología, 33(11), 1066-1079.

Justel, N., O'Conor, J. \& Rubinstein, W. (2015). Modulación de la memoria emocional a través de la música en adultos mayores: Un estudio preliminar [Modulation of emotional memory through music in older adults: A preliminary study]. Interdisciplinaria, 32(2), 247-259. http: //dx.doi.org/10.16888/interd.2015.32.2.3

Kennelly, J. \& Edwards, J. (1997). Providings music therapy to the unconscious child in the paediatric intensive care unit. Australian Journal of Music Therapy, 8, 18-29.

Koelsch, E. (2010). Towards a neural basis of music-evoked emotions. Trends in Cognitive Sciences, 14(3), 131-137. http://doi.org/10.1016/j. tics.2010.01.002

LaPuma, J., Schiedermayer, D.L., Culyas, A.E. \& Siegler, M. (1988). Talking to comatose patients. Archives of Neurology, 45, 20-22.

Machado, C. (2007). Cerebral response to patient's own name in the vegetative and minimally conscious states. Neurology, 68, 895-899.

Magee, W.L. (2005). Music therapy with patients in low awareness states: Approaches to assessment and treatment in multidisciplinary care. Neuropsychological Rehabilitation: An International Journal, 15(4), 522-536.

Magee, W.L. (2007). Development of a music therapy assessment tool for patients in low awareness states. NeuroRehabilitation, 22(4), 319-324.

Magee, W.L., Ghetti, C.L \& Moyer, A. (2015). Feasibility of the music therapy assessment tool for awareness in disorders of consciousness (MATADOC) for use with pediatric populations. Frontiers in Psychology, 6(698), 1-12.

Magee, W. L. \& O'Kelly, J. (2014). Music therapy with disorders of consciousness: Current evidence and emergent evidence-based practice. Poster presentado en The Neurosciences and Music V, Dijon, France.

Magee, W.L. \& O'Kelly, J. (2015). Music therapy with disorders of consciousness: current evidence and emergent evidence-based practice. Annals of the New York Academy of Sciences, 
1337, 256-262. http://doi.org/10.1111/nyas.1 2633

McKinney, C.H., Antoni, M.H., Kumar M, Tims, F.C. \& McCabe, P.M. (1997). Effects of guided imagery and music (GIM) therapy on mood and cortisol in healthy adults. Health Psychology, 16, 390-400.

Mitchell, S., Bradley, V. A., Welch, J.L. \& Britton, P.G. (1990). Coma arousal procedure: A therapeutic intervention in the treatment of head injury. Bain Injury, 4(3), 273-279.

Noé Sebastián, E., Moliner Muñoz, B., O’Valle Rodríguez, M., Balmaseda Serrano, R., Colomer Front, C., Navarro Pérez, M.D. \& Ferri Campos, J. (2012). Historia y humanidades. Del estado vegetativo al estado de vigilia sin respuesta: Una revisión histórica [History and humanities. From vegetative state to waking state without response: A historical review]. Revista de Neurología, 55(5), 306-313.

O'Callaghan, C. (1999). Recent findings about neural correlates of music pertinent to music therapy across the lifespan. Music Therapy Perspectives, 17(1), 32-36.

O’Kelly, J., James, L., Palaniappan, R., Taborin, J., Fachner, J. \& Magee, W. (2013). Neurophysiological and behavioral responses to music therapy in vegetative and minimally conscious states. Frontiers in Human Neuroscience, 7(884). http://doi.org/10.3389/fnhum.2013.00884

O’Kelly, J. \& Magee, E. (2013a). Music therapy with disorders of consciousness and neuroscience: The need for dialogue. Nordic Journal of Music Therapy, 22, 93-106.

O'Kelly, J. \& Magee, E. (2013b). The complementary role of music therapy in the detection of awareness in disorders of consciouness: An audit of concurrent SMART and MATADOC assesments. Neuropsychological Rehabilitation, 23, 287-298.
Papousek, H. (1996). Musicality in infancy research: Biological and cultural origins of early musicality. En I. Deliege \& J. Sloboda (Eds.), Musical beginnings: Origins and development of musical competence (pp. 37-55). Oxford: Oxford University Press.

Peretz, I. \& Zatorre, R.J. (2005). Brain organization for music processing. Annual Review of Psychology, 56, 89-114. http://doi.org/10.1146/an nurev.psych.56.091103.070225

Plum, F. \& Posner, J. (1980). The diagnosis of stupor and coma. Philadelphia: PA:FA Davis.

Ponsford, J., Sloan, S. \& Snow, P. (1995). Traumatic brain injury: In rehabilitation for every day adaptive living. London: Lawrence Erlbaum.

Purves, D., Augustine, G.J., Fitzpatrick, D., Hall, W.C., Lamantia, A.S., McNamara, J.O. \& Williams, S.M. (2007). Neurociencia [Neuroscience]. Buenos Aires, Argentina: Médica Panamericana.

Rader, M.A. \& Ellis, D.W. (1989). Sensory stimulation assesment measure: Manual for administration. Camden, NJ: Institute of Brain Injury Research and Teaching.

Raglio, A., Guizzetti, G.B., Bolognesi, M., Antonaci, D., Granieri, E., Baiardi, P. et al. (2014). Active music therapy approach in disorders of consciousness: a controlled observational case series. Journal of Neurology, 261, 2460-2462. http://doi.org/10.1007/s00415-014-7543-0

Rappaport, M., Dougherty, A.M. \& Kelting, D.L. (1992). Evaluation of coma and vegetative states. Archives of Physical Medicine and Rehabilitation, 73, 628-634.

Rollnik, J. \& Altenmüller, E. (2014). Music in disorders of consciousness. Frontiers in Neuroscience, 8(3), 1-6. http://doi.org/10. 3389/fnins. 2014.00190

Rosenfeld, J. \& Dun, B. (1999). Music therapy in children with severe traumatic brain injury. En 
R.R. Pratt \& D. Grocke (Eds.), MusicMedicine3. MusicMedicine and music therapy: Expanding horizons (pp. 35-46). Melbourne: Faculty of Music, University of Melbourne.

Royal College of Physicians. (2013). Prolonged disorders of consciousness: National clinical guidelines. London: RCP.

Schnakers, C. (2009). Detecting consciousness in a total locked-in syndrome: An active eventrelated paradigm. Neurocase, 15, 271-277.

Shifres, F. (2014). Algo más sobre el enlace entre la infancia temprana y la música: el poder expresivo del rubato. En S. Español (Ed.), Psicología de la música y del desarrollo. Una exploración interdisciplinaria sobre la musicalidad humana [Psychology of music and development. An interdisciplinary exploration of human musicality]. Buenos Aires, Argentina: Paidós.

Sloboda, J. (1991a). Music structure and emotional response: Some empirical findings. Psychology of Music, 19, 110-120.

Sloboda, J. (1991b). Empirical studies of emotional response to music. En M.R. Jones \& S. Holleran (Eds.), Cognitive bases of musical communication (pp. 33-46). Washington: APA.

Tamplin, J. (2000). Improvisational music therapy approches to coma arousal. Australian Journal of Music Therapy, 11, 38-51.

Thaut, M. (2005). Rhythm, music and the brain: Scientific foundations and clinical applications. New York: Routledge.
Thaut, M. \& Hoemberg, V. (2014). Handbook of neurologic music therapy. Oxford: Oxford University Press.

Trevarthen, C. (1999). Musicality and the instrinsic motive pulse: Evidence from human psychobiology and infant communication in rhythms, musical narrative and origins of musical communication. Musicae Scientia, 3, 155-215.

Trevarthen, C. (2002). Origins of musical identity: Evidence from infancy for musical social awareness. En R. MacDonald, D.J. Hargreaves \& D. Miell (Eds.), Musical identities (pp. 21-38). Oxford: Oxford University Press.

Vogl, J., Heine, A,, Steinhoff, N., Weiss, K. \& Tucek, G. (2015). Neuroscientific and neuroanthropological perspectives in music therapy research and practice with patients with disorders of consciousness. Frontier Neuroscience, 9, 273. http://doi.org/10.3389/fnins.2015.002 73

Wainsztein, N. \& Leiguarda, R. (2005). Neurología [Neurology]. Argentina: El Ateneo.

Wilson, S. L., Powell, G. E., Brock, D. \& Thwaites, H. (1996). Behavioural differences between patiens who emerged from vegetative state and those who did not. Brain Injury, 10(7), 509-516.

World Federation of Music Therapy. (2011). Recuperado el 3 de septiembre de 2015 de http://ww w.musictherapyworld.net

Laboratorio de Psicología Experimental y Aplicada (PSEA) Instituto de Investigaciones Médicas (IDIM) Consejo Nacional de Investigaciones Científicas y Técnicas (CONICET)

Universidad de Buenos Aires (UBA) Ciudad Autónoma de Buenos Aires República Argentina

Fecha de recepción: 18 de febrero de 2016 Fecha de aceptación: 19 de agosto de 2016 